Bull. Korean Math. Soc. 48 (2011), No. 2, pp. 291-302

DOI 10.4134/BKMS.2011.48.2.291

\title{
RELATIONS AMONG THE FIRST VARIATION, THE CONVOLUTIONS AND THE GENERALIZED FOURIER-GAUSS TRANSFORMS
}

\author{
Man Kyu Im, Un Cig Ji, and Yoon Jung Park
}

\begin{abstract}
We first study the generalized Fourier-Gauss transforms of functionals defined on the complexification $\mathcal{B}_{\mathbf{C}}$ of an abstract Wiener space $(\mathcal{H}, \mathcal{B}, \nu)$. Secondly, we introduce a new class of convolution products of functionals defined on $\mathcal{B}_{\mathbf{C}}$ and study several properties of the convolutions. Then we study various relations among the first variation, the convolutions, and the generalized Fourier-Gauss transforms.
\end{abstract}

\section{Introduction}

In [1], Cameron introduced a transform of functionals defined on the complexification $K_{0}[0, T]$ of the Wiener space $C_{0}[0, T]$ which is called the FourierWiener transform, and later it was modified by Cameron and Martin in [3]. More precisely, for a functional $F$ defined on $K_{0}[0, T]$, the Fourier-Wiener transform $\mathcal{G}_{1, i} F$ of $F$ is defined by

$$
\mathcal{G}_{1, i} F(y)=\int_{C_{0}[0 . T]} F(x+i y) m(d x), \quad y \in K_{0}[0, T]
$$

whenever it exists, where $m$ is the Wiener measure, and the modified FourierWiener transform $\mathcal{G}_{\sqrt{2}, i} F$ of a functional $F$ defined on $K_{0}[0, T]$ is defined by

$$
\mathcal{G}_{\sqrt{2}, i} F(y)=\int_{C_{0}[0 . T]} F(\sqrt{2} x+i y) m(d x), \quad y \in K_{0}[0, T]
$$

if the integral exists. Also, in [7], Gross introduced $\nu_{t}$-convolution defined by

$$
\left(\nu_{t} F\right)(y)=\mathcal{G}_{\sqrt{t}, 1} F(y)=\int_{\mathcal{B}} F(\sqrt{t} x+y) \nu(d x)
$$

Received June 26, 2009; Revised October 23, 2009.

2010 Mathematics Subject Classification. Primary 28C20; Secondary 44A20, 44A35.

Key words and phrases. abstract Wiener space, generalized Fourier-Gauss transform, convolution, first variation.

This work was supported by the Korea Science and Engineering Foundation (KOSEF) grant funded by the Korea government (MEST) (No. R01-2008-000-10843-0). 
for certain functional $F$ defined on $\mathcal{B}$, where $(\mathcal{H}, \mathcal{B}, \nu)$ is an abstract Wiener space, and then Lee in [15] introduced an integral transform $\mathcal{G}_{\alpha, \beta} F$ of a functional $F$ defined on the complexification $\mathcal{B}_{\mathbf{C}}$ of the abstract Wiener space $(\mathcal{H}, \mathcal{B}, \nu)$ and then $\mathcal{G}_{\alpha, \beta} F$ is called the Fourier-Gauss transform (see, [5, 9, 14] and the references are cited there in) of $F$ which is defined by

$$
\mathcal{G}_{\alpha, \beta} F(y)=\int_{\mathcal{B}} F(\alpha x+\beta y) \nu(d x), \quad y \in \mathcal{B}_{\mathbf{C}}
$$

whenever it exists, where $\alpha, \beta \in \mathbf{C}$. In [5], the Fourier-Gauss transform was generalized by Chung and Ji in white noise distribution theory (see, $[8,14,16]$ ) which, in the abstract Wiener space, can be defined by

$$
\mathcal{G}_{A, B} F(y)=\int_{\mathcal{B}} F(A x+B y) \nu(d x), \quad y \in \mathcal{B}_{\mathbf{C}}
$$

whenever it exists, where $A$ and $B$ are continuous linear operators from $\mathcal{B}_{\mathbf{C}}$ into itself, and called the generalized Fourier-Gauss transform. Recently, in [12], Ji and Obata investigated that the generalized Fourier-Gauss transforms play an important role for the Bogoliubov transforms.

On the other hand, Yeh in [17] introduced a convolution product $F_{1} * F_{2}$ of functionals $F_{1}$ and $F_{2}$ defined on $K_{0}[0, T]$ by

$$
\left(F_{1} * F_{2}\right)(y)=\int_{C_{0}[0, T]} F_{1}\left(\frac{x+y}{\sqrt{2}}\right) F_{2}\left(\frac{x-y}{\sqrt{2}}\right) m(d x), \quad y \in K_{0}[0, T]
$$

whenever it exists, and studied an interesting relation between the FourierWiener transform and the convolution product:

$$
\mathcal{G}_{1, i}\left(F_{1} * F_{2}\right)(x)=\left(\mathcal{G}_{1, i} F_{1}\right)(y / \sqrt{2})\left(\mathcal{G}_{1, i} F_{2}\right)(-y / \sqrt{2}), \quad y \in K_{0}[0, T]
$$

for functionals $F_{1}$ and $F_{2}$ in a certain class of functionals defined on $K_{0}[0, T]$. Then the Yeh's results were extended to the abstract Wiener space by Yoo in [18]. For the study of various relations between the convolution product and the analytic Fourier-Feynman transform, we refer to $[4,10]$.

Main purposes of this paper are three folds. We first study the generalized Fourier-Gauss transform of functionals defined on $\mathcal{B}_{\mathbf{C}}$ (see Section 3 ). Secondly, we introduce a new class of convolutions $F_{1} *_{A, B, C, D} F_{2}$ of functionals $F_{1}$ and $F_{2}$ defined on $\mathcal{B}_{\mathbf{C}}$ which is defined by

$$
\left(F_{1} *_{A, B, C, D} F_{2}\right)(x)=\int_{\mathcal{B}} F_{1}(A x+B y) F_{2}(C x+D y) \nu(d y), \quad x \in \mathcal{B}_{\mathbf{C}},
$$

whenever the integral exists, where $A, B, C, D$ are continuous linear operators from $\mathcal{B}_{\mathbf{C}}$ into itself, and then we study several properties of the convolutions (see Section 4). Finally, we study various relations among the first variation, the convolutions and the generalized Fourier-Gauss transforms (see Section 5).

The study of several relations between the first variation, the convolutions and the analytic Fourier-Feynman transform are now in progress. 


\section{Abstract Wiener space}

In this section, we shall briefly recall of concepts, notations and known results in the abstract Wiener space $[7,13]$. Let $(\mathcal{H}, \mathcal{B}, \nu)$ be an abstract Wiener space, i.e., $\mathcal{H}$ is a real (separable) Hilbert space, $\mathcal{B}$ is a real Banach space which is the completion of $\mathcal{H}$ with respect to a weaker (measurable) norm $\|\cdot\|_{\mathcal{B}}$ than the norm on the Hilbert norm, and $\nu$ is the standard Gaussian measure on $\mathcal{B}$. The strong dual space of $\mathcal{B}$ is denoted by $\mathcal{B}^{*}$. Let $\mathcal{B}_{\mathbf{C}}=\{x+i y \mid x, y \in \mathcal{B}\}$ be the complexification of $\mathcal{B}$. For each $x \in \mathcal{B}, h \in \mathcal{B}^{*},\langle x, h\rangle$ is well-defined Gaussian random variable with mean 0 and variance $\|h\|_{\mathcal{H}}^{2}$, where $\langle\cdot, \cdot\rangle$ is the complex bilinear form on $\mathcal{B}_{\mathbf{C}} \times \mathcal{B}_{\mathbf{C}}^{*}$.

For each $m \geq 0$, let $\mathcal{E}_{a}(m)$ be the class of functions $\phi$ on $\mathcal{B}_{\mathbf{C}}$ satisfying that

(i) for each $x, y \in \mathcal{B}_{\mathbf{C}}, \phi(x+\lambda y)$ is an entire function of $\lambda \in \mathbf{C}$;

(ii) the norm

$$
\|\phi\|_{m}=\sup _{x \in \mathcal{B}_{\mathbf{C}}}|\phi(x)| \exp \left\{-m\|x\|_{\mathcal{B}_{\mathbf{C}}}\right\}
$$

is finite, where $\|\cdot\|_{\mathcal{B}_{\mathbf{C}}}$ is the norm on $\mathcal{B}_{\mathbf{C}}$.

For each $\xi$ in $\mathcal{B}_{\mathbf{C}}^{*}$, consider the function $e^{\langle\cdot, \xi\rangle}$ defined on $\mathcal{B}_{\mathbf{C}}$. Then

$$
\left|e^{\langle x, \xi\rangle}\right| \leq e^{\|x\|_{\mathcal{B}_{\mathbf{C}}}\|\xi\|}
$$

and so for any $m \geq\|\xi\|$

$$
\left\|e^{\langle\cdot, \xi\rangle}\right\|_{m} \leq \sup _{x \in \mathcal{B}_{\mathbf{C}}} e^{(\|\xi\|-m)\|x\|_{\mathcal{B}_{\mathbf{C}}}} \leq 1
$$

and $e^{\langle\cdot, \xi\rangle}$ in $\mathcal{E}_{a}(m)$.

For each $0 \leq m \leq n$, we have

$$
\|\phi\|_{n}=\sup _{x \in \mathcal{B}_{\mathbf{C}}}|\phi(x)| e^{-n\|x\|_{\mathcal{B}_{\mathbf{C}}}} \leq \sup _{x \in \mathcal{B}_{\mathbf{C}}}|\phi(x)| e^{-m\|x\|_{\mathcal{B}_{\mathbf{C}}}} \leq\|\phi\|_{m}
$$

and so, $\mathcal{E}_{a}(m) \subset \mathcal{E}_{a}(n)$. Put

$$
\mathcal{E}_{a} \equiv \operatorname{ind}_{m \rightarrow \infty} \lim _{a} \mathcal{E}_{a}(m) .
$$

Then for any $\xi \in \mathcal{B}_{\mathbf{C}}^{*}, e^{\langle\cdot, \xi\rangle} \in \mathcal{E}_{a}$ and the exponential vector (or coherent state) $\phi_{\xi}$ is defined by

$$
\phi_{\xi}=e^{\langle\cdot, \xi\rangle-\frac{1}{2}\langle\xi, \xi\rangle} .
$$

Then $\phi_{\xi} \in \mathcal{E}_{a}$ and $\left\{\phi_{\xi} \mid \xi \in \mathcal{B}_{\mathbf{C}}^{*}\right\}$ spans a dense subspace of $\mathcal{E}_{a}$, see Corollary 3.3 .8 in [16].

Theorem 2.1 (Fernique Theorem). There exists $\beta>0$ such that

$$
\int_{\mathcal{B}} \exp \left(\beta\|x\|_{\mathcal{B}_{\mathbf{C}}}^{2}\right) \nu(d x)<\infty .
$$

By Theorem 2.1, the following corollary is immediate.

Corollary 2.2. $\int_{\mathcal{B}} \exp \left(\beta\|x\|_{\mathcal{B}_{\mathbf{C}}}\right) \nu(d x)<\infty$ for all $\beta>0$. 


\section{Generalized Fourier-Gauss transform}

Let $\mathcal{L}\left(\mathcal{B}_{\mathbf{C}}, \mathcal{B}_{\mathbf{C}}\right)$ denote the space of all continuous linear operators on $\mathcal{B}_{\mathbf{C}}$. Let $S$ and $T$ be in $\mathcal{L}\left(\mathcal{B}_{\mathbf{C}}, \mathcal{B}_{\mathbf{C}}\right)$. Now, we study the generalized Fourier-Gauss transform introduced in [5].

Definition 3.1. Let $\phi$ be a functional defined on $\mathcal{B}_{\mathbf{C}}$ and $S, T \in \mathcal{L}\left(\mathcal{B}_{\mathbf{C}}, \mathcal{B}_{\mathbf{C}}\right)$. Then the generalized Fourier-Gauss transform $\mathcal{G}_{S, T} \phi$ of $\phi$ is defined by

$$
\mathcal{G}_{S, T} \phi(x) \equiv \int_{\mathcal{B}} \phi(S x+T y) \nu(d y), \quad x \in \mathcal{B}_{\mathbf{C}}
$$

whenever it exists.

Note that for each $S, T \in \mathcal{L}\left(\mathcal{B}_{\mathbf{C}}, \mathcal{B}_{\mathbf{C}}\right), \phi \in \mathcal{E}_{a}(m)$ and $x \in \mathcal{B}_{\mathbf{C}}$, we have

$$
\int_{\mathcal{B}}|\phi(S x+T y)| \nu(d y) \leq\|\phi\|_{m} e^{m\|S\|\|x\|_{\mathcal{B}_{\mathbf{C}}}} \int_{\mathcal{B}}\left(e^{m\|T\|\|y\|_{\mathcal{B}}}\right) \nu(d y)
$$

and then by Corollary 2.2, the last integral is finite. Therefore, for each $x \in \mathcal{B}_{\mathbf{C}}$, the integral $\int_{\mathcal{B}} \phi(S x+T y) \nu(d y)$ exists, and so the generalized Fourier-Gauss transform $\mathcal{G}_{S, T} \phi$ of $\phi \in \mathcal{E}_{a}(m)$ is well-defined.

Theorem 3.2. Let $S, T \in \mathcal{L}\left(\mathcal{B}_{\mathbf{C}}, \mathcal{B}_{\mathbf{C}}\right)$. Then for each $m, n \geq 0$ such that $m\|S\| \leq n$, the generalized Fourier-Gauss transform $\mathcal{G}_{S, T}$ is continuous linear from $\mathcal{E}_{a}(m)$ into $\mathcal{E}_{a}(n)$.

Proof. For each $\phi \in \mathcal{E}_{a}(m)$, we have

$$
\begin{aligned}
\left\|\mathcal{G}_{S, T} \phi\right\|_{n} & =\sup _{x \in \mathcal{B}_{\mathbf{C}}}\left|\mathcal{G}_{S, T} \phi(x)\right| e^{-n\|x\|_{\mathcal{B}_{\mathbf{C}}}} \\
& \leq \sup _{x \in \mathcal{B}_{\mathbf{C}}}\left(\int_{\mathcal{B}}\|\phi\|_{m}\left(e^{m\|S x+T y\|_{\mathcal{B}_{\mathbf{C}}}}\right) \nu(d y)\right) e^{-n\|x\|_{\mathcal{B}_{\mathbf{C}}}} \\
& \leq\|\phi\|_{m}\left(\sup _{x \in \mathcal{B}_{\mathbf{C}}} e^{(m\|S\|-n)\|x\|_{\mathcal{B}_{\mathbf{C}}}}\right) \int_{\mathcal{B}}\left(e^{m\|T\|\|y\|_{\mathcal{B}_{\mathbf{C}}}}\right) \nu(d y) .
\end{aligned}
$$

Since $m\|S\|-n \leq 0$,

$$
\left\|\mathcal{G}_{S, T} \phi\right\|_{n} \leq\left(\int_{\mathcal{B}}\left(e^{m\|T\|\|y\|_{\mathcal{B}_{\mathbf{C}}}}\right) \nu(d y)\right)\|\phi\|_{m}
$$

and so, by Corollary $2.2, \mathcal{G}_{S, T}$ is continuous from $\mathcal{E}_{a}(m)$ into $\mathcal{E}_{a}(n)$.

Theorem 3.3. Let $S, T \in \mathcal{L}\left(\mathcal{B}_{\mathbf{C}}, \mathcal{B}_{\mathbf{C}}\right)$. The generalized Fourier-Gauss transform $\mathcal{G}_{S, T}$ is continuous linear from $\mathcal{E}_{a}$ into itself.

Proof. The proof is immediate by Theorem 3.2.

Proposition 3.4. For each $S, T \in \mathcal{L}\left(\mathcal{B}_{\mathbf{C}}, \mathcal{B}_{\mathbf{C}}\right)$ and $\phi_{\xi} \in \mathcal{E}_{a}$, the generalized Fourier-Gauss transform $\mathcal{G}_{S, T} \phi_{\xi}$ of $\phi_{\xi}$ is given by

$$
\mathcal{G}_{S, T} \phi_{\xi}(x)=e^{\left\langle x, S^{*} \xi\right\rangle+\frac{1}{2}\left\langle\left(T T^{*}-I\right) \xi, \xi\right\rangle}=e^{\frac{1}{2}\left\langle\left(S S^{*}+T T^{*}-I\right) \xi, \xi\right\rangle} \phi_{S^{*} \xi}(x) .
$$

Proof. The proof is straightforward. 


\section{Convolutions and generalized Fourier-Gauss transforms}

Let $A, B, C, D \in \mathcal{L}\left(\mathcal{B}_{\mathbf{C}}, \mathcal{B}_{\mathbf{C}}\right)$. We introduce a new class of convolutions of functionals on $\mathcal{B}_{\mathbf{C}}$, and study various relations between the convolutions and the generalized Fourier-Gauss transforms.

Definition 4.1. Let $\phi$ and $\psi$ be functionals defined on $\mathcal{B}_{\mathbf{C}}$. Then the convolution $\phi *_{A, B, C, D} \psi$ of $\phi$ and $\psi$ is defined by

$$
\phi *_{A, B, C, D} \psi(x) \equiv \int_{\mathcal{B}} \phi(A x+B y) \psi(C x+D y) \nu(d y), \quad x \in \mathcal{B}_{\mathbf{C}}
$$

whenever it exists.

Lemma 4.2. Let $m_{1}, m_{2} \geq 0$. For each $\phi \in \mathcal{E}_{a}\left(m_{1}\right), \psi \in \mathcal{E}_{a}\left(m_{2}\right)$, the convolution $\phi *_{A, B, C, D} \psi(x)$ is well-defined.

Proof. For each $\phi \in \mathcal{E}_{a}\left(m_{1}\right), \psi \in \mathcal{E}_{a}\left(m_{2}\right)$, we obtain that

$$
|\phi(A x+B y)| \leq\|\phi\|_{m_{1}} e^{m_{1}\|A x+B y\|_{\mathcal{B}_{\mathbf{C}}}} \leq\|\phi\|_{m_{1}} e^{m_{1}\|A\|\|x\|_{\mathcal{B}_{\mathbf{C}}}+m_{1}\|B\|\|y\|_{\mathcal{B}_{\mathbf{C}}}}
$$

and

$$
|\psi(C x+D y)| \leq\|\psi\|_{m_{2}} e^{m_{2}\|C\|\|x\|_{\mathcal{B}_{\mathbf{C}}}+m_{2}\|D\|\|y\|_{\mathcal{B}_{\mathbf{C}}}} .
$$

Therefore, we have

$$
\begin{aligned}
& \int_{\mathcal{B}}|\phi(A x+B y) \| \psi(C x+D y)| \nu(d y) \\
\leq & \|\phi\|_{m_{1}}\|\psi\|_{m_{2}} e^{\left(m_{1}\|A\|+m_{2}\|C\|\right)\|x\|_{\mathcal{B}_{\mathbf{C}}}} \int_{\mathcal{B}} e^{\left(m_{1}\|B\|+m_{2}\|D\|\right)\|y\|_{\mathcal{B}}} \nu(d y) .
\end{aligned}
$$

Since by Corollary 2.2 the last integral as in (4.2) is finite, the convolution $\phi *_{A, B, C, D} \psi$ is well-defined.

Corollary 4.3. For each $\phi, \psi \in \mathcal{E}_{a}$, the convolution $\phi *_{A, B, C, D} \psi$ is well-defined.

Proof. The proof is immediate by Lemma 4.2 .

Theorem 4.4. The convolution $*_{A, B, C, D}$ is separately continuous from $\mathcal{E}_{a} \times \mathcal{E}_{a}$ into $\mathcal{E}_{a}$.

Proof. For each $m, m_{1}, m_{2} \geq 0$ with $m \geq m_{1}\|A\|+m_{2}\|C\|$ and $\phi \in \mathcal{E}_{a}\left(m_{1}\right)$, $\psi \in \mathcal{E}_{a}\left(m_{2}\right)$, we have

$$
\begin{aligned}
& \left\|\phi *_{A, B, C, D} \psi\right\|_{m} \\
= & \sup _{x \in \mathcal{B}_{\mathbf{C}}}\left|\int_{\mathcal{B}} \phi(A x+B y) \psi(C x+D y) \nu(d y)\right| e^{-m\|x\|_{\mathcal{B}_{\mathbf{C}}}} \\
\leq & \sup _{x \in \mathcal{B}_{\mathbf{C}}}\|\phi\|_{m_{1}}\|\psi\|_{m_{2}} e^{\left(m_{1}\|A\|+m_{2}\|C\|-m\right)\|x\|_{\mathcal{B}_{\mathbf{C}}}} \int_{\mathcal{B}} e^{\left(m_{1}\|B\|+m_{2}\|D\|\right)\|y\|_{\mathcal{B}_{\mathbf{C}}} \nu(d y)} \\
\leq & \left(\int_{\mathcal{B}} e^{\left.\left(m_{1}\|B\|+m_{2}\|D\|\right)\|y\|_{\mathcal{B}_{\mathbf{C}}} \nu(d y)\right)\|\phi\|_{m_{1}}\|\psi\|_{m_{2}}}\right.
\end{aligned}
$$

as desired. 
Proposition 4.5. For each $\phi_{\xi}, \phi_{\eta} \in \mathcal{E}_{a}$, the convolution $\phi_{\xi} *_{A, B, C, D} \phi_{\eta}$ has the following form:

(4.3)

$$
\phi_{\xi} *_{A, B, C, D} \phi_{\eta}(x)=e^{\left\langle x, A^{*} \xi+C^{*} \eta\right\rangle+\left\langle D B^{*} \xi, \eta\right\rangle+\frac{1}{2}\left\langle\left(B B^{*}-I\right) \xi, \xi\right\rangle+\frac{1}{2}\left\langle\left(D D^{*}-I\right) \eta, \eta\right\rangle} .
$$

Proof. The proof is straightforward. In fact, we obtain that

$$
\begin{aligned}
\left(\phi_{\xi} * A, B, C, D \phi_{\eta}\right)(x) & =e^{-\frac{1}{2}\langle\xi, \xi\rangle-\frac{1}{2}\langle\eta, \eta\rangle+\left\langle x, A^{*} \xi+C^{*} \eta\right\rangle} \int_{\mathcal{B}} e^{\left\langle y, B^{*} \xi+D^{*} \eta\right\rangle} \nu(d y) \\
& =e^{\left\langle x, A^{*} \xi+C^{*} \eta\right\rangle+\frac{1}{2}\left\langle B^{*} \xi+D^{*} \eta, B^{*} \xi+D^{*} \eta\right\rangle-\frac{1}{2}\langle\xi, \xi\rangle-\frac{1}{2}\langle\eta, \eta\rangle} \\
& =e^{\left\langle x, A^{*} \xi+C^{*} \eta\right\rangle+\left\langle D B^{*} \xi, \eta\right\rangle+\frac{1}{2}\left\langle\left(B B^{*}-I\right) \xi, \xi\right\rangle+\frac{1}{2}\left\langle\left(D D^{*}-I\right) \eta, \eta\right\rangle}
\end{aligned}
$$

as desired.

Theorem 4.6. The convolution $*_{A, B, C, D}$ is a commutative operation, i.e., $\phi *_{A, B, C, D} \psi=\psi *_{A, B, C, D} \phi$ for $\phi, \psi \in \mathcal{E}_{a}$ if and only if

$$
A=C, \quad B B^{*}=D D^{*}, \quad D B^{*}=B D^{*} \text {. }
$$

Moreover, if $A, B, C, D$ are constants, then (4.4) is equivalent to that $A=C$, $B^{2}=D^{2}$

Proof. The proof is straightforward from Theorem 4.4 and Proposition 4.5.

A relation between the convolution and the generalized Fourier-Gauss transform is studied in Theorem 4.7.

Theorem 4.7. Let $S_{i}, T_{i}, A_{j}, B_{j}, C_{j}, D_{j} \in \mathcal{L}\left(\mathcal{B}_{\mathbf{C}}, \mathcal{B}_{\mathbf{C}}\right), i=1,2,3, j=1,2$. Then for any $\phi, \psi \in \mathcal{E}_{a}$,

$$
\mathcal{G}_{S_{1}, T_{1}}\left(\phi *_{A_{1}, B_{1}, C_{1}, D_{1}} \psi\right)(x)=\left(\mathcal{G}_{S_{2}, T_{2}} \phi *_{A_{2}, B_{2}, C_{2}, D_{2}} \mathcal{G}_{S_{3}, T_{3}} \psi\right)(x)
$$

if and only if the following conditions are satisfied:

(i) $A_{1} S_{1}=S_{2} A_{2}, C_{1} S_{1}=S_{3} C_{2}$;

(ii) $\left(A_{1} T_{1}\right)\left(A_{1} T_{1}\right)^{*}+B_{1} B_{1}^{*}=\left(S_{2} B_{2}\right)\left(S_{2} B_{2}\right)^{*}+T_{2} T_{2}^{*}$;

(iii) $\left(C_{1} T_{1}\right)\left(C_{1} T_{1}\right)^{*}+D_{1} D_{1}^{*}=\left(S_{3} D_{2}\right)\left(S_{3} D_{2}\right)^{*}+T_{3} T_{3}^{*}$;

(iv) $\left(C_{1} T_{1}\right)\left(A_{1} T_{1}\right)^{*}+D_{1} B_{1}^{*}=\left(S_{3} D_{2}\right)\left(S_{2} B_{2}\right)^{*}$.

Proof. For each $\xi, \eta \in \mathcal{B}_{\mathbf{C}}^{*}$, by (4.3) and (3.1) we have

$$
\begin{aligned}
& \mathcal{G}_{S_{1}, T_{1}}\left(\phi_{\xi} * A_{1}, B_{1}, C_{1}, D_{1} \phi_{\eta}\right)(x) \\
= & e^{\left\langle x,\left(A_{1} S_{1}\right)^{*} \xi+\left(C_{1} S_{1}\right)^{*} \eta\right\rangle} \\
& \times e^{\frac{1}{2}\left[\left\langle\left(A_{1} T_{1}\left(A_{1} T_{1}\right)^{*}+B_{1} B_{1}^{*}-I\right) \xi, \xi\right\rangle+\left\langle\left(C_{1} T_{1}\left(C_{1} T_{1}\right)^{*}+D_{1} D_{1}^{*}-I\right) \eta, \eta\right\rangle\right]} \\
& \times e^{\left\langle\left(C_{1} T_{1}\left(A_{1} T_{1}\right)^{*}+D_{1} B_{1}^{*}\right) \xi, \eta\right\rangle}
\end{aligned}
$$

and

$$
\begin{aligned}
& \left(\mathcal{G}_{S_{2}, T_{2}} \phi_{\xi} * A_{2}, B_{2}, C_{2}, D_{2} \mathcal{G}_{S_{3}, T_{3}} \phi_{\eta}\right)(x) \\
= & e^{\left\langle x,\left(S_{2} A_{2}\right)^{*} \xi+\left(S_{3} C_{2}\right)^{*} \eta\right\rangle}
\end{aligned}
$$




$$
\begin{aligned}
& \times e^{\frac{1}{2}\left[\left\langle\left(S_{2} B_{2}\left(S_{2} B_{2}\right)^{*}+T_{2} T_{2}^{*}-I\right) \xi, \xi\right\rangle+\left\langle\left(S_{3} D_{2}\left(S_{3} D_{2}\right)^{*}+T_{3} T_{3}^{*}-I\right) \eta, \eta\right\rangle\right]} \\
& \times e^{\left\langle S_{3} D_{2}\left(S_{3} B_{2}\right)^{*} \xi, \eta\right\rangle} .
\end{aligned}
$$

Therefore, by comparing (4.6) and (4.7) the proof is straightforward.

From Theorem 4.7, the following corollary is immediate.

Corollary 4.8. Let $S, T, A_{j}, B_{j}, C_{j}, D_{j} \in \mathcal{L}\left(\mathcal{B}_{\mathbf{C}}, \mathcal{B}_{\mathbf{C}}\right), j=1,2$. Then for any $\phi, \psi \in \mathcal{E}_{a}$,

$$
\mathcal{G}_{S, T}\left(\phi *_{A_{1}, B_{1}, C_{1}, D_{1}} \psi\right)=\left(\mathcal{G}_{S, T} \phi\right) *_{A_{2}, B_{2}, C_{2}, D_{2}}\left(\mathcal{G}_{S, T} \psi\right)
$$

if and only if the following conditions are satisfied:

(i) $A_{1} S=S A_{2}, C_{1} S=S C_{2}$;

(ii) $\left(A_{1} T\right)\left(A_{1} T\right)^{*}+B_{1} B_{1}^{*}=\left(S B_{2}\right)\left(S B_{2}\right)^{*}+T T^{*}$;

(iii) $\left(C_{1} T\right)\left(C_{1} T\right)^{*}+D_{1} D_{1}^{*}=\left(S D_{2}\right)\left(S D_{2}\right)^{*}+T T^{*}$;

(iv) $\left(C_{1} T\right)\left(A_{1} T\right)^{*}+D_{1} B_{1}^{*}=\left(S D_{2}\right)\left(S B_{2}\right)^{*}$.

Corollary 4.9. Suppose that $*_{A_{i}, B_{i}, C_{i}, D_{i}}, i=1,2$, are commutative operations. Then for any $\phi, \psi \in \mathcal{E}_{a}$,

$$
\mathcal{G}_{S, T}\left(\phi *_{A_{1}, B_{1}, C_{1}, D_{1}} \psi\right)=\left(\mathcal{G}_{S, T} \phi\right) *_{A_{2}, B_{2}, C_{2}, D_{2}}\left(\mathcal{G}_{S, T} \psi\right)
$$

if and only if the following conditions are satisfied:

(i) $A_{1} S=S A_{2}$;

(ii) $\left(A_{1} T\right)\left(A_{1} T\right)^{*}+B_{1} B_{1}^{*}=\left(S B_{2}\right)\left(S B_{2}\right)^{*}+T T^{*}$;

(iii) $\left(C_{1} T\right)\left(A_{1} T\right)^{*}+D_{1} B_{1}^{*}=\left(S D_{2}\right)\left(S B_{2}\right)^{*}$.

Proof. Suppose that $*_{A_{i}, B_{i}, C_{i}, D_{i}}$ are commutative convolutions. Then by Theorem 4.6, the conditions (ii) and (iii) in Corollary 4.8 are equivalent, and the condition (i) in Corollary 4.8 is simplified by (i). Therefore, the proof is immediate form Corollary 4.8.

Corollary 4.10. Let $S, T, A_{j}, B_{j}, C_{j}, D_{j}$ be complex numbers. Then (4.8) holds for any $\phi, \psi \in \mathcal{E}_{a}$ if and only if the following conditions are satisfied:

(i) $A_{1} S=A_{2} S, C_{1} S=C_{2} S$;

(ii) $A_{1}^{2} T^{2}+B_{1}^{2}=S^{2} B_{2}^{2}+T^{2}$;

(iii) $C_{1}^{2} T^{2}+D_{1}^{2}=S^{2} D_{2}^{2}+T^{2}$

(iv) $A_{1} C_{1} T^{2}+B_{1} D_{1}=S^{2} B_{2} D_{2}$.

In particular, we study the case of $B_{2}=D_{2}=0$ which implies that the convolution $*_{A_{2}, B_{2}, C_{2}, D_{2}}$ coincides with the composition of pointwise product and dilations.

Corollary 4.11. Let $S, T, A_{j}, B_{j}, C_{j}, D_{j}$ be complex numbers and $B_{2}=D_{2}=$ 0 . Then (4.8) holds for any $\phi, \psi \in \mathcal{E}_{a}$ if and only if the following conditions are satisfied:

(i) $A_{1} S=A_{2} S, C_{1} S=C_{2} S$;

(ii) $A_{1}^{2} T^{2}+B_{1}^{2}=T^{2}$; 
(iii) $C_{1}^{2} T^{2}+D_{1}^{2}=T^{2}$;

(iv) $A_{1} C_{1} T^{2}+B_{1} D_{1}=0$.

Corollary 4.12. Let $B_{2}=D_{2}=0$ and let $S, T \in \mathbf{C}$ with $S \neq 0$ and $T \neq 0$. Suppose that $0 \neq B_{1} \in \mathbf{C}$ and $A_{1}=\alpha \in \mathbf{C}$. Then (4.8) holds for any $\phi, \psi \in \mathcal{E}_{a}$ if and only if

(4.9) $A_{2}=A_{1}=\alpha, \quad B_{1}^{2}=\left(1-\alpha^{2}\right) T^{2}, C_{1}^{2}=1-\alpha^{2}, \quad C_{2}=C_{1}, \quad D_{1}^{2}=\alpha^{2} T^{2}$.

Proof. Equation (4.8) holds for any $\phi, \psi \in \mathcal{E}_{a}$ if and only if the conditions (i)-(iv) in Corollary 4.11 holds, and then (i) implies that

$$
A_{2}=A_{1}, \quad C_{2}=C_{1}
$$

since $S \neq 0$. By (iii) and (iv), since $T \neq 0$, the condition $B_{1} \neq 0$ implies that $C_{1} \neq 0$ and so (iv), (ii) and (iii) imply that

$$
\frac{A_{1}}{B_{1}} T^{2}=-\frac{D_{1}}{C_{1}}, \quad \frac{A_{1}^{2}}{B_{1}^{2}} T^{2}=\frac{T^{2}}{B_{1}^{2}}-1, \quad T^{2}+\frac{D_{1}^{2}}{C_{1}^{2}}=\frac{T^{2}}{C_{1}^{2}} .
$$

Therefore, we have

$$
\frac{T^{2}}{C_{1}^{2}}=\frac{D_{1}^{2}}{C_{1}^{2}}+T^{2}=\frac{A_{1}^{2}}{B_{1}^{2}} T^{4}+T^{2}=\frac{\alpha^{2}}{B_{1}^{2}} T^{4}+T^{2}
$$

which implies that

$$
C_{1}^{2}=\frac{B_{1}^{2}}{\alpha^{2} T^{2}+B_{1}^{2}} .
$$

On the other hand, by (ii) we have

$$
B_{1}^{2}=\left(1-A_{1}^{2}\right) T^{2}=\left(1-\alpha^{2}\right) T^{2}
$$

and so

$$
C_{1}^{2}=\frac{\left(1-\alpha^{2}\right) T^{2}}{\alpha^{2} T^{2}+\left(1-\alpha^{2}\right) T^{2}}=1-\alpha^{2} .
$$

Also, by (iii) and (4.12) we have

$$
D_{1}^{2}=\left(1-C_{1}^{2}\right) T^{2}=\alpha^{2} T^{2} .
$$

Hence by (4.10), (4.11), (4.12) and (4.13), we obtain (4.9). The proof of the converse is straightforward.

Example 4.13. The convolution $* \equiv *_{A, B, C, D}$ with $A=B=D=\frac{1}{\sqrt{2}}$ and $C=-\frac{1}{\sqrt{2}}$ has been introduced in [17]. Also, it is obvious that $\phi * \frac{1}{\sqrt{2}}, 0,-\frac{1}{\sqrt{2}}, 0, \psi$ coincides with $\phi\left(\frac{1}{\sqrt{2}} \cdot\right) \psi\left(-\frac{1}{\sqrt{2}} \cdot\right)$ and then by Corollary 4.11 , we have

$$
\mathcal{G}(\phi * \psi)(z)=\mathcal{G} \phi\left(\frac{z}{\sqrt{2}}\right) \mathcal{G} \psi\left(-\frac{z}{\sqrt{2}}\right),
$$


where $\mathcal{G} \equiv \mathcal{G}_{i, 1}$ is the Fourier-Wiener transform, which is one of the main results in [17] and a special case $S=i, T=1$ and $\alpha=1 / \sqrt{2}$ of Corollary 4.12. Moreover, by Corollary 4.12 , for any $0 \neq S \in \mathbf{C}$ and $\phi, \psi \in \mathcal{E}_{a}$, we have

$$
\mathcal{G}_{S, 1}(\phi * \psi)(z)=\mathcal{G}_{S, 1} \phi\left(\frac{z}{\sqrt{2}}\right) \mathcal{G}_{S, 1} \psi\left(-\frac{z}{\sqrt{2}}\right) .
$$

Corollary 4.14. Suppose that $S, T, A_{i}, B_{i}, C_{i}, D_{i}, i=1,2$, are complex numbers and the convolutions $*_{A_{i}, B_{i}, C_{i}, D_{i}}, i=1,2$, are commutative operations. Then

$$
\mathcal{G}_{S, T}\left(\phi *_{A_{1}, B_{1}, C_{1}, D_{1}} \psi\right)=\left(\mathcal{G}_{S, T} \phi\right) *_{A_{2}, B_{2}, C_{2}, D_{2}}\left(\mathcal{G}_{S, T} \psi\right)
$$

if and only if the following conditions are satisfied:

(i) $A_{1} S=A_{2} S$;

(ii) $A_{1}^{2} T^{2}+B_{1}^{2}=B_{2}^{2} S^{2}+T^{2}$;

(iii) $A_{1}^{2} T^{2}+B_{1} D_{1}=S^{2} B_{2} D_{2}$.

The proof is immediate from Corollary 4.9.

\section{First variation, convolutions and transforms}

Let $\mathcal{D}=\operatorname{LS}\left\{\phi_{\xi} \mid \xi \in \mathcal{B}_{\mathbf{C}}^{*}\right\}$, where $\operatorname{LS}(X)$ means the linear span of $X$. In this section, we study various relationships among the three concepts of generalized Fourier-Gauss transform, convolution, and first variation for functionals belong to $\mathcal{D}$.

Definition 5.1. Let $\phi$ be a functional defined on $\mathcal{B}_{\mathbf{C}}$ and $w \in \mathcal{B}_{\mathbf{C}}$. Then the first variation $\delta \phi(\cdot \mid w)$ of $\phi$ in the direction $w$ is defined by

$$
\delta \phi(y \mid w)=\left.\frac{\partial}{\partial t} \phi(y+t w)\right|_{t=0}, \quad y \in \mathcal{B}_{\mathbf{C}}
$$

whenever it exists.

Theorem 5.2. Let $S, T \in \mathcal{L}\left(\mathcal{B}_{\mathbf{C}}, \mathcal{B}_{\mathbf{C}}\right)$ and $w \in \mathcal{B}_{\mathbf{C}}$. Then for any $\phi \in \mathcal{D}$

$$
\mathcal{G}_{S, T}(\delta \phi(y \mid S w))=\delta\left(\mathcal{G}_{S, T} \phi\right)(y \mid w), \quad y \in \mathcal{B}_{\mathbf{C}}
$$

Proof. For $y \in \mathcal{B}_{\mathbf{C}}$ and $\xi \in \mathcal{B}_{\mathbf{C}}^{*}$, we have

$$
\mathcal{G}_{S, T}\left(\delta \phi_{\xi}(y \mid S w)\right)=\mathcal{G}_{S, T}\left(\langle S w, \xi\rangle \phi_{\xi}\right)(y)=\langle S w, \xi\rangle \mathcal{G}_{S, T} \phi_{\xi}(y),
$$

where we use the fact that $\delta \phi_{\xi}(y \mid w)=\langle w, \xi\rangle \phi_{\xi}(y)$ for any $\xi \in \mathcal{B}_{\mathbf{C}}^{*}$ and $w \in \mathcal{B}_{\mathbf{C}}$. On the other hand, by (3.1) we have

$$
\delta\left(\mathcal{G}_{S, T} \phi_{\xi}\right)(y \mid w)=\left\langle w, S^{*} \xi\right\rangle \mathcal{G}_{S, T} \phi_{\xi}(y)=\langle S w, \xi\rangle \mathcal{G}_{S, T} \phi_{\xi}(y) .
$$

Therefore, by (5.16) and (5.17), we have (5.15).

Theorem 5.3. Let $A, B, C, D \in \mathcal{L}\left(\mathcal{B}_{\mathbf{C}}, \mathcal{B}_{\mathbf{C}}\right)$ and $w \in \mathcal{B}_{\mathbf{C}}$. Then for each $\phi, \psi \in \mathcal{E}_{a}$ such that $\frac{\partial}{\partial t} \phi(x+t w)$ and $\frac{\partial}{\partial t} \psi(x+t w)$ are bounded by an integrable function in $x$ with respect to the measure $\nu$, we have

$(5.18) \delta\left(\phi *_{A, B, C, D} \psi\right)(y \mid w)=\left(\delta \phi(\cdot \mid A w) *_{A, B, C, D} \psi+\phi *_{A, B, C, D} \delta \psi(\cdot \mid C w)\right)(y)$ 
for $y \in \mathcal{B}_{\mathbf{C}}$.

Proof. Let $y \in \mathcal{B}_{\mathbf{C}}$. Then for each $\phi, \psi \in \mathcal{E}_{a}$ such that $\frac{\partial}{\partial t} \phi(x+t w)$ and $\frac{\partial}{\partial t} \psi(x+t w)$ are bounded by an integrable function in $x$ with respect to the measure $\nu$, by applying Lebesgue convergence theorem we obtain that

$$
\begin{aligned}
& \delta\left(\phi *_{A, B, C, D} \psi\right)(y \mid w) \\
= & \left.\int_{\mathcal{B}} \frac{\partial}{\partial t}[\phi(A(y+t w)+B z) \psi(C(y+t w)+D z)]\right|_{t=0} \nu(d z) \\
= & \left.\int_{\mathcal{B}} \frac{\partial \phi(A y+B z+t A w)}{\partial t}\right|_{t=0} \psi(C y+D z) \nu(d z) \\
& +\left.\int_{\mathcal{B}} \phi(A y+B z) \frac{\partial \psi(C y+D z+t C w)}{\partial t}\right|_{t=0} \nu(d z) \\
= & {\left[\delta \phi(\cdot \mid A w) *_{A, B, C, D} \psi\right](y)+\left[\phi *_{A, B, C, D} \delta \psi(\cdot \mid C w)\right](y), }
\end{aligned}
$$

which implies (5.18).

In Theorem 5.3, we study the derivation property of the first variation with respect to the convolutions. In fact, we consider $D_{w} \phi=\delta \phi(\cdot \mid w), \phi \in \mathcal{D}$, then $D_{w}$ has the derivative property with respect to the convolution if and only if $w=A w=C w$.

A relation between the generalized Fourier-Gauss transform and the the convolution is studied in the following theorem.

Theorem 5.4. We keep notations and assumptions as in Theorem 4.7. Let $w \in \mathcal{B}_{\mathbf{C}}$. Then for any $\phi, \psi \in \mathcal{D}$,

$$
\begin{aligned}
& \delta \mathcal{G}_{S_{1}, T_{1}}\left(\phi *_{A_{1}, B_{1}, C_{1}, D_{1}} \psi\right)(y \mid w) \\
= & {\left[\mathcal{G}_{S_{2}, T_{2}} \delta \phi\left(\cdot \mid S_{1} A_{2} w\right) *_{A_{2}, B_{2}, C_{2}, D_{2}} \mathcal{G}_{S_{3}, T_{3}} \psi\right](y) } \\
& +\left[\mathcal{G}_{S_{2}, T_{2}} \phi *_{A_{2}, B_{2}, C_{2}, D_{2}} \mathcal{G}_{S_{3}, T_{3}} \delta \psi\left(\cdot \mid S_{3} C_{2} w\right)\right](y) .
\end{aligned}
$$

Proof. The proof is straightforward by applying Theorems 4.7, 5.3 and 5.2.

In the next corollary, we study a intertwining property of the generalized Fourier-Gauss transform and the first variation. For the more study of the intertwining property, we refer to $[5,6]$.

Corollary 5.5. Let $A, B \in \mathcal{L}\left(\mathcal{B}_{\mathbf{C}}, \mathcal{B}_{\mathbf{C}}\right)$. Then for any $\phi \in \mathcal{D}$,

$$
\delta\left(\mathcal{G}_{A, B} \phi\right)(y \mid w)=\mathcal{G}_{A, B}(\delta \phi(y \mid A w)), \quad y \in \mathcal{B}_{\mathbf{C}} .
$$

Proof. By Theorem 5.4, we have

$$
\delta\left(\mathcal{G}_{A, B} \phi\right)(y \mid w)=\delta\left(\phi *_{A, B, C, D} \mathbf{1}\right)(y \mid w)=\mathcal{G}_{A, B}(\delta \phi(y \mid A w)), \quad y \in \mathcal{B}_{\mathbf{C}}
$$

which implies (5.19). 


\section{References}

[1] R. H. Cameron, Some examples of Fourier-Wiener transforms of analytic functionals, Duke Math. J. 12 (1945), 485-488.

[2] R. H. Cameron, The first variation of an indefinite Wiener integral, Proc. Amer. Math. Soc. 2 (1951), 914-924.

[3] R. H. Cameron and W. T. Martin, Fourier-Wiener transforms of analytic functionals, Duke Math. J. 12 (1945), 489-507.

[4] R. H. Cameron and D. A. Storvick, An $L_{2}$ analytic Fourier-Feynman transform, Michigan Math. J. 23 (1976), no. 1, 1-30.

[5] D. M. Chung and U. C. Ji, Transforms on white noise functionals with their applications to Cauchy problems, Nagoya Math. J. 147 (1997), 1-23.

[6] _ Transformation groups on white noise functionals and their applications, Appl. Math. Optim. 37 (1998), no. 2, 205-223.

[7] L. Gross, Potential theory on Hilbert space, J. Funct. Anal. 1 (1967), 123-181.

[8] T. Hida, Analysis of Brownian Functionals, Carleton Mathematical Lecture Notes, No. 13. Carleton Univ., Ottawa, Ont., 1975.

[9] T. Hida, H. H. Kuo, and N. Obata, Transformations for white noise functionals, J. Funct. Anal. 111 (1993), no. 2, 259-277.

[10] T. Huffman, C. Park, and D. Skoug, Analytic Fourier-Feynman transforms and convolution, Trans. Amer. Math. Soc. 347 (1995), no. 2, 661-673.

[11] U. C. Ji and N. Obata, Quantum white noise calculus, Non-commutativity, infinitedimensionality and probability at the crossroads, 143-191, QP-PQ: Quantum Probab. White Noise Anal., 16, World Sci. Publ., River Edge, NJ, 2002.

[12] _ Bogoliubov transformations in terms of generalized Fourier-Gauss transformations, preprint, 2008.

[13] H. H. Kuo, Gaussian Measures in Banach Spaces, Lecture Notes in Mathematics, Vol. 463. Springer-Verlag, Berlin-New York, 1975.

[14] White Noise Distribution Theory, CRC Press, Boca Raton, FL, 1996.

[15] Y. J. Lee, Integral transforms of analytic functions on abstract Wiener spaces, J. Funct. Anal. 47 (1982), no. 2, 153-164.

[16] N. Obata, White Noise Calculus and Fock Space, Lecture Notes in Mathematics, 1577. Springer-Verlag, Berlin, 1994.

[17] J. Yeh, Convolution in Fourier-Wiener transform, Pacific J. Math. 15 (1965), 731-738.

[18] I. Yoo, Convolution and the Fourier-Wiener transform on abstract Wiener space, Rocky Mountain J. Math. 25 (1995), no. 4, 1577-1587.

MAN KYU IM

Department of Mathematics

HANNAM UNIVERSITY

DAEJEON 306-791, KoreA

E-mail address: mki@hannam.ac.kr

UN Cig Ji

Department of Mathematics

Research institute of Mathematical Finance

Chungbuk National University

Cheonguu 361-763, Korea

E-mail address: uncigji@chungbuk.ac.kr 
YoOn Jung PARK

Department of Mathematics

Chungbuk National University

Cheongue 361-763, Korea

E-mail address: yjpark@chungbuk.ac.kr 